\title{
Nebulizer induced superinfection and sepsis with Chryseobacterium indologenes in a postoperative patient with Acinetobacter baumannii pneumonia: a case report and review
}

\author{
Kanne Padmaja ${ }^{1}$, Vemu Lakshmi', Yelliboina Sreekanth ${ }^{2}$, Ramachandran Gopinath ${ }^{2}$ \\ 'Department of Microbiology Nizam's Institute of Medical Sciences, Hyderabad, AP, India \\ ${ }^{2}$ Department of Anesthesiology, Nizam's Institute of Medical Sciences, Hyderabad, AP, India
}

doi: 10.3396/ijic.v8i2.019.12

\begin{abstract}
Chryseobacterium indologenes, a non-fermentative Gram-negative bacillus, is an uncommon pathogen in humans causing a variety of invasive infections, especially in hospitalized patients with severe underlying disease and with indwelling devices. We herein report a case of superinfection and sepsis caused by $C$. indologenes in a postoperative patient being managed in the respiratory intensive care unit (RICU) for acute respiratory distress syndrome and Acinetobacter baumannii infection. C. indologenes was probably acquired from an ultrasonic nebulizer used for colistin aerosolization against $A$. baumannii. Subsequently the patient developed superinfection and sepsis due to $C$. indologenes. The patient had a favourable response to a prolonged therapy with fluoroquinolones and trimethoprim sulfamethoxazole.
\end{abstract}

Key words

NEBULIZERS AND VAPORIZERS; ACINETOBACTER BAUMANNII; INTENSIVE CARE UNITS; SEPSIS microbiology; BACTEREMIA - microbiology

\section{Introduction}

Chryseobacterium species are uncommon human pathogens found in the environment and cause variety of infections, usually in patients with altered immune status or in infants. ${ }^{1}$ The organisms are ubiquitous in nature and widely distributed in soil, plants, and water. ${ }^{2}$
In the hospital environments, they have been recovered from water systems, humid surfaces and wet medical devices (such as ventilators, tubes and humidifiers) and serve as potential reservoirs of infection. ${ }^{3}$ Chryseobacterium species, have been documented as human pathogens causing a variety of invasive

\section{Corresponding author}

Dr V Lakshmi

Department of Microbiology, Nizam's Institute of Medical Sciences,

Panjagutta, Hyderabad - 500082, Andhra Pradesh, India.

Email: vemulakshmigorthi@gmail.com | Tel.No. 9140 66618773, 914023489290 
infections, especially in hospitalized patients with severe underlying disease and on indwelling devices. ${ }^{4}$

We hereby report a case of $C$. indologenes catheter related blood stream infection (CRBSI) with sepsis in a postoperative patient on ventilatory support.

\section{Case Report}

This is a case of $C$. indologenes superinfection with sepsis in a 35 years female patient who presented with jejunal perforation and underwent jejunal bypass. In the postoperative period, she developed fever, bilateral crepitations with worsening respiratory distress which progressed to acute respiratory distress syndrome (ARDS). She was then shifted to RICU for ventilatory support for the ARDS. A percutaneous tracheostomy was performed for ventilation. Cultures of the tracheal aspirate sent on the $5^{\text {th }}$ postoperative day to the microbiology laboratory, yielded significant growth of Acinetobacter baumannii, sensitive only to colistin. She received inhalation colistin through a nebulizer on the $9^{\text {th }}$ postoperative day, for three consecutive days. In spite of these measures the patient continued to have persistent fever. Though there were signs of basal pneumonia, the chest radiograph did not show any new infiltrates.

Complete blood picture showed elevated leukocytosis. The surgical wound was sterile and well healed. Repeat culture of the tracheal aspirate, on the $13^{\text {th }}$ day, showed significant growth of $C$. indologenes, susceptible to cotrimoxazole, ciprofloxacin, levofloxacin and tetracycline. Blood cultures (2 sets of standard aerobic and FAN BacT/alert bottles, bioMerieux, Marcy-IEtoile,France) also yielded the same organism with similar susceptibility pattern. A. baumannii was not isolated from any of the specimen. Patient was started on intravenous ciprofloxacin 100mg 12th hourly and oral cotrimoxazole (TMP-SMX) 500mg 6th hourly. However, fever was persistent and continuous and repeat blood cultures (2 sets each from the peripheral veins and central venous catheter) on day $22^{\text {nd }}$ yielded C. indologenes with a similar susceptibility as the earlier isolate. There was no evidence of right sided endocarditis on $2 \mathrm{D}$ ECHO. The indwelling CVC was removed and was sent for culture to the microbiology laboratory. C. indologenes was found on the CVC. The same treatment was continued for the next 6 weeks.
Patient responded to this prolonged antibiotic therapy and gradually the fever and lung condition improved and she was weaned off from the ventilator. She was later shifted out of the RICU to a general ward and was discharged after 10 days. At discharge, she was afebrile, her lungs were clear and the surgical scar was well healed and repeat blood cultures were sterile.

\section{Microbiology workup}

The isolate from the tracheal aspirates and blood cultures was a non fastidious Gram negative coccobacillus and grew on both 5\% sheep blood agar and chrome agar (COS \& CPS, bioMerieux, Marcy l' Etoile, France) aerobically at $37^{\circ} \mathrm{C}$ by $24 \mathrm{hrs}$. Morphologically, the colonies were $1-2 \mathrm{~mm}$ smooth, circular, moist distinct orange to yellow pigmentation, on both the plates (figure 1). The organism was oxidase and catalase positive and could be accurately identified as $C$. indologenes with ID-GN panel of the Vitek 2 system (bioMerieux, Marcy l' Etoile, France) as a non-fermentor. However, the mini API (bioMerieux, Marcy $l^{\prime}$ Etoile, France) failed to identify the isolate accurately. Antimicrobial susceptibility testing was performed with N090 panel of the Vitek 2 system. The same strain of $C$. indologenes was also isolated from the central venous catheter (using Maki's method of semiquantitative cultures ${ }^{5}$ ) and swab cultures from the inlet and outlet of the nebulizer, through which colistin was administered to the patient. However, molecular strain typing for relatedness was not attempted.

\section{Discussion}

The genus Chryseobacterium, formerly known as Flavobacterium, was first defined in 1994 by Vandamme et al. and is described in Bergey's Manual of Systematic Bacteriology. ${ }^{6}$ The 6 species of the genus Chryseobacterium that are more commonly isolated from clinical specimen are C. meningosepticum, $C$. odoratum, C. multivorum, C. breve and group $11 \mathrm{~b}$ Chryseobacterium spp, which includes C. indologenes and C. gleum. ${ }^{7}$ Chryseobacteria are exogenous human pathogens and are found in the water systems, surface of equipment and wet medical devices within the hospital. ${ }^{7}$ These opportunistic pathogens can cause a variety of infections such as bacteraemia, ventilatorassociated pneumonia, indwelling device-associated infection, pyelonephritis, biliary tract infection, peritonitis, lumboperitoneal shunt infection, ocular 


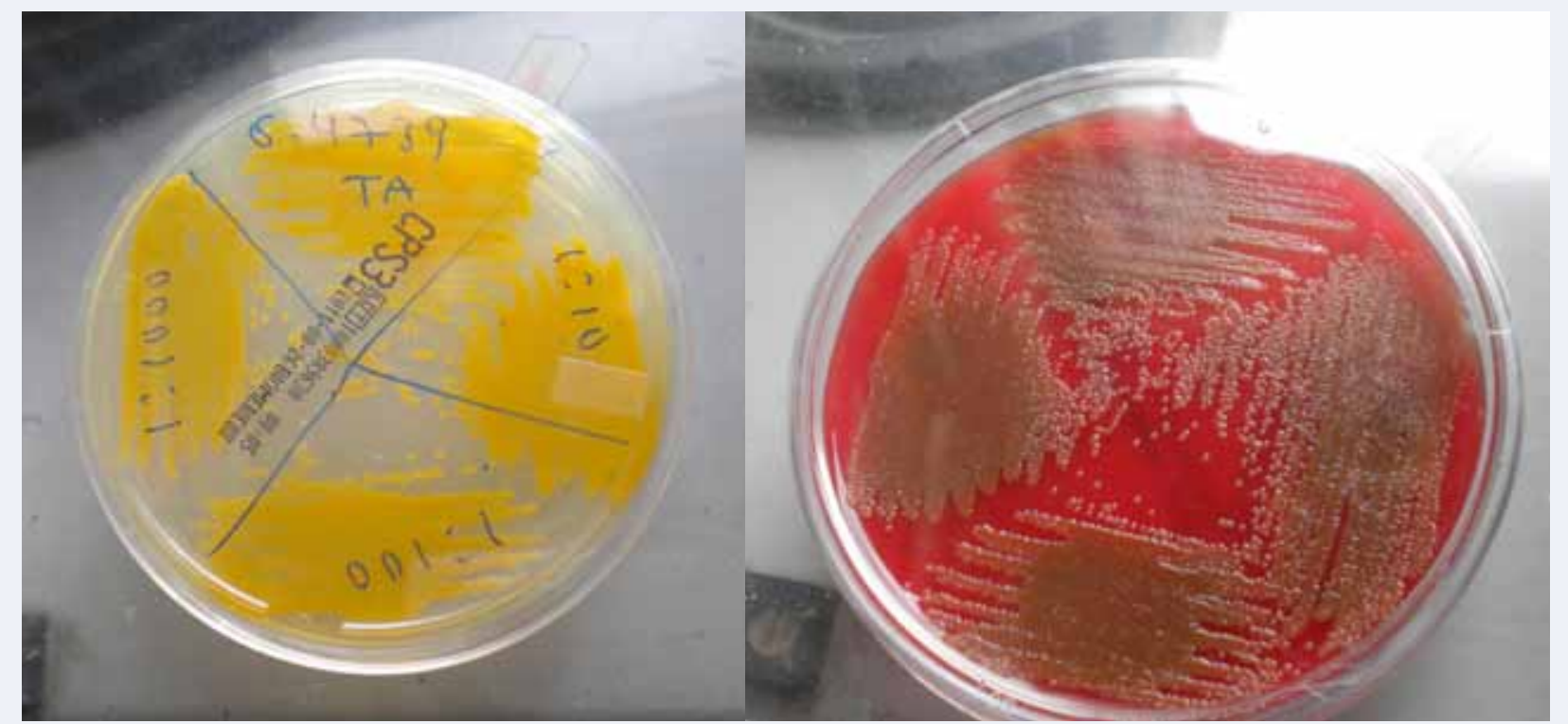

Figure 1. Colony morphology on chrome agar (COS \& CPS, bioMerieux, Marcy l' Etoile, France) and $5 \%$ sheep blood agar

infections, surgical and burn wound infections. ${ }^{1,7}$ In our case the surgical wound was sterile and did not yield any organism.

C. indologenes is an uncommon human pathogen and has been isolated from the tracheal aspirate of patients with ventilator-associated pneumonia. ${ }^{7}$ The majority of infections caused by $C$. indologenes have been reported from Taiwan, which include 48 cases of bacteraemia and 6 of these were in children. ${ }^{7} \mathrm{~A}$ recent case report from India showed that $C$. indologenes was isolated from blood cultures of a pre-term baby who was on ventilatory support in the ICU. ${ }^{8}$ The risk factors for infections with $C$. indologenes include underlying medical illness like malignancies and diabetes mellitus, long-term treatment with broad-spectrum antibiotics and presence of indwelling devices. Approximately half of $C$. indologenes infections have been shown to be associated with indwelling devices that include intravascular catheters, surgical drainage, feeding tubes, endotracheal tubes and Foley's catheters. ${ }^{9}$ Production of biofilms on these foreign materials and protease activity of the bacterium may play an important role in the virulence and invasiveness of this pathogen. ${ }^{4}$

In the present case, the source of the organism was probably from the nebulizer which later progressed to blood stream infection, as was evidenced by the growth of the organism from the nebulizer and peripheral blood culture on the $13^{\text {th }}$ day. However, the source of contamination of the nebulizer is not known and could probably be at the point of use. The indwelling central venous catheter was probably later colonised with $C$. indolegenes, from the blood stream and was a source of prolonged CVCRBSI. There was no evidence of CVC exit site infection.

C. indologenes is a colistin resistant organism. The optimal choice of an effective antibiotic for $C$. indologenes infection is challenging as the organism has a limited spectrum of antimicrobial sensitivity. According to the SENTRY antimicrobial surveillance programme, the most effective drugs against $C$. indologenes are the quinolones ( $>95 \%$ susceptibility), TMP-SMX (>95\% susceptibility) and piperacillintazobactam (>90\%susceptibility). Though other effective agents are ceftazidime, cefepime and rifampin ( $>80 \%$ susceptibility), ${ }^{3,4,10}$ our isolate showed in vitro resistance to the betalactams, piperacillin-tazobactum and aminoglycosides. In addition to an effective antibiotic therapy, removal of the indwelling catheter should be performed if clinical symptoms persist, as was done in our case.

In conclusion, $\mathrm{C}$. indologenes should be considered as a potential opportunistic pathogen in the ICU setting. Fluoroquinolones and TMP-SMX are the most appropriate antimicrobial agents as also seen in our patient with a good response and favourable outcome 
with these agents. ${ }^{11}$ Resistance to a wide range of antimicrobial agents such as aminoglycosides, extended-spectrum penicillins, third and fourth generation cephalosporins, chloramphenicol, linezolid and glycopeptides has been documented and had very little effect or no effect against this pathogen. Therefore choosing an appropriate antibiotic therapy for this pathogen is very difficult. ${ }^{12}$ Strict infection control measures including care of the humidifiers should be in place to prevent such infections with uncommon pathogens like the $C$. indologenes. Epidemiological, virulence and pathogenicity factors of this uncommon pathogen have to be further studied.

\section{References}

1. Douvoyiannis M, Kalyoussef S, Philip G, Mayers MM. Chryseobacterium indologenes Bacteraemia in an infant. Int J Infect Dis 2010; 14: 531-532. http://dx.doi.org/10.1016/j. ijid.2009.06.015

2. Bayraktar MR, Aktas E, Ersoy Y, Cicek A, Durnaz R. Postoperative Chryseobacterium indologenes blood stream infections caused by contamination of distilled water. Infect Control Hosp Epidemiol 2007; 28: 368-369. http://dx.doi. org/10.1086/508839

3. Ferreira RS, Brandão FFB, Lobo SM. Chryseobacterium indologenes infection: a case report. Rev Bras Ter Intensiva 2010; 22: 96-198. http://dx.doi.org/10.1590/S0103$507 X 2010000100016$

4. Christakis GB, Perlorentzou SP, Chalkiopoulou I, Athanasiou A, Legakis NJ. Chryseobacterium indologenes non-catheterrelated bacteremia in a patient with a solid tumor. I Clin Microbiol 2005; 43(4): 2021-2023. http://dx.doi.org/10.1128/ JCM.43.4.2021-2023.2005
5. Maki DG, Weise CE, Sarafin HW. A semiquantitative culture method for identifying intravenous catheter related infection. NEJM 1977; 296: 1305 - 1309. http://dx.doi.org/10.1056/ NEJM197706092962301

6. Vandamme P, Bernardet JF, Segers P, Kersters K, Holmes B. New Perspectives in the Classification of the Flavobacteria: Description of Chryseobacterium gen.nov. Bergeyella gen. nov., and Empedobacter nom.rev. Int / Syst Bacteriol 1994; 44: 827-831. http://dx.doi.org/10.1099/00207713-44-4-827

7. Calderón G, García E, Rojas P, García E, Rosso M, Losada A. Chryseobacterium indologenes infection in a newborn: a case report. J Medical Case Reports 2011; 5: 10. http://dx.doi. org/10.1186/1752-1947-5-10

8. Sudharani V, Asiya, Saxena NK. Chryseobacterium indologenes bacteraemia in a preterm baby. Ind J Med Microbiol 2011; 29: 196-198. http://dx.doi.org/10.4103/0255-0857.81783

9. Hsueh PR, Teng LJ, Ho SW, Hsueh WC, Luh KT. Clinical and microbiological characteristics of Flavobacterium indologenes infections associated with indwelling devices. J Clin Microbio 1996; 34: 1908-1913.

10. Kirby JT, Sader HS, Walsh TR, Jones RL. Antimicrobial susceptibility and epidemiology of a world wide collection of Chryseobacterium spp: Report from the SENTRY. Antimicrobial Surveillance Program (1997-2001). J Clin Microbiol 2004; 42: 445-448. http://dx.doi.org/10.1128/ JCM.42.1.445-448.2004

11. Lin YT, Jeng YY, Lin ML, Yu KW, Wang FD, Liu CY. Clinical and Microbiologocal Characteristics of Chryseobacterium indologenes Bacteraemia. I Microbiol Immunology and Infection 2010; 6: 498-505. http://dx.doi.org/10.1016/S16841182(10)60077-1

12. Chow DW, Wu SL, Lee CT, Tai FT, Yu WL. Clinical Characteristics, Antimicrobial Susceptibilities, and Outcomes of Patients with Chryseobacterium indologenes Bacteremia in an Intensive Care Unit. Japanese Journal of Infectious Diseases 2011; 64(6): 520-524. 